\title{
Ion Induced Bidirectional Bending for Controlled Manipulation at Nanoscale
}

Vivek Garg ${ }^{1,2,3}$, Rakesh G. Mote ${ }^{2 *}$, Tsengming Chou ${ }^{4}$, Amelia Liu ${ }^{5}$, Alex De Marco ${ }^{6}$, Bhaveshkumar Kamaliya ${ }^{1,2,3}$, Shi Qiu ${ }^{3}$, Jing $\mathrm{Fu}^{3 *}$

1. IITB-Monash Research Academy, Indian Institute of Technology Bombay, Mumbai, India.

2. Department of Mechanical Engineering, Indian Institute of Technology Bombay, Mumbai, India.

3. Department of Mechanical and Aerospace Engineering, Monash University, Melbourne, Australia.

4. Laboratory of Multiscale Imaging, Stevens Institute of Technology, Hoboken, NY, USA.

5. Monash Centre for Electron Microscopy, Monash University, Melbourne, Australia.

6. Department of Biochemistry and Molecular Biology, Monash University, Melbourne, Australia.

* Corresponding authors: rakesh.mote@iitb.ac.in, jing.fu@monash.edu

Ion irradiation and implantation are used extensively for inducing functionalities and engineering of nanostructures for diverse applications in the nanotechnology domain. Nanostructures, under ion irradiations, exhibit distinct transformations in contrast to their bulk counterparts due to the nanostructure dimensions comparable to collision cascade. Tensile, compressive stresses can be developed in the lattice atoms under ion irradiations, inducing plastic deformation, which in turn can be used for controlled manipulation at nanoscale, thus providing opportunities for extended functionality and strain engineering of nanostructures. Silicon (Si) nanostructures, with a high index and large band gap, offer unique light-matter interactions for optical applications such as color filtering etc. [1], [2] Conventional lithography methods such as electron beam lithography (EBL), however, consist of multiple steps requiring mask preparation, resist development, dry/wet etching etc. and the experimental realization of $3 \mathrm{D}$, complex features for extended functionalities becomes especially challenging due to the design intricacy, with limitations on the feature size and aspect ratio.

In this work, a novel method for fabrication of functional 3D nanostructures through wet etching of ion implanted Si for freestanding nanostructures is developed, followed by in-situ kiloelectronvolt ion irradiation induced bidirectional bending [3] for controlled manipulation at nanoscale in a dual beam FIB-SEM microscope. Wet etching of ion implanted Si allows realization of freestanding nanostructures such as Si NW (Fig. 1a). To investigate the underlying physics and ion induced deformation mechanism of Si nanostructures, Si nanowires are investigated, which are found to deform bi-directionally in-situ by controlled irradiation of ions (Fig. 1b, c). Experimental studies on ion irradiation induced bending provide new insights for controlling nanostructures in-situ, and it was found that the NWs bent in the downward direction initially and reverses its bending direction beyond a threshold ion dose $\left(\sim 7 \times 10^{14}\right.$ ions $/ \mathrm{cm}^{2}$ ) eventually aligning towards incident ion beam at $30 \mathrm{kV}$. Such bidirectional bending of nanostructures is associated with the implanted-ions, generation of point defects, and dislocated lattice atoms, in addition to the sputtering effects at high ion doses, contributing to the generation of localized stresses developing ion irradiation induced plastic deformation. Further, controlled manipulation of nanostructures through ion induced strain-engineering is utilized for new nanoscale configurations and applications. An array of vertically bent Si NWs is shown through SEM image in Fig. 1d, while Fig. 1e shows a NW bent in two alternative directions. Various nanostructure configurations can be realized through tuning of ion-dose and energy, providing an additional degree of freedom and extending ion beam application for realization of new functional 3D nanostructures. Fig. 1 (f) includes SEM image of a curved 3D mesh nanostructure demonstrating the controlled manipulation. The developed approach for fabrication of various suspended and 3D structures exhibit process capability and will be useful for 
fabrication of Si micro/nano-mechanical resonators, sensors, and semiconductor devices. In summary, successful demonstration of nanostructure fabrication and controlled manipulation at nanoscale through in-situ ion irradiation provide a new approach for 3D nanofabrication and realization of unique geometries having potential for development of future nanoscale devices and applications beyond material science in the diverse field of ion beams [4].

\section{References:}

[1] V Garg, RG Mote, and J Fu, Advance Materials Technologies 3 (2018), p. 1800100.

[2] V Garg, RG Mote, and J Fu, Microscopy and Microanalysis 24 (2018), p. 856.

[3] V Garg et al., Weaving Nanostructures with Site-Specific Ion Induced Bidirectional Bending (under review).

[4] The work is financially supported by IITB-Monash Research Academy, the Australian Research Council (DP180103955), IRCC (Seed grant: Spons/ME/I14079-1/2014), IIT Bombay, and Tata Consultancy Services (TCS) research scholarship program. The authors acknowledge the use of facilities within the Monash Centre for Electron Microscopy (MCEM), and Melbourne Centre for Nanofabrication (MCN), Victorian Node of the Australian National Fabrication Facility (ANFF).

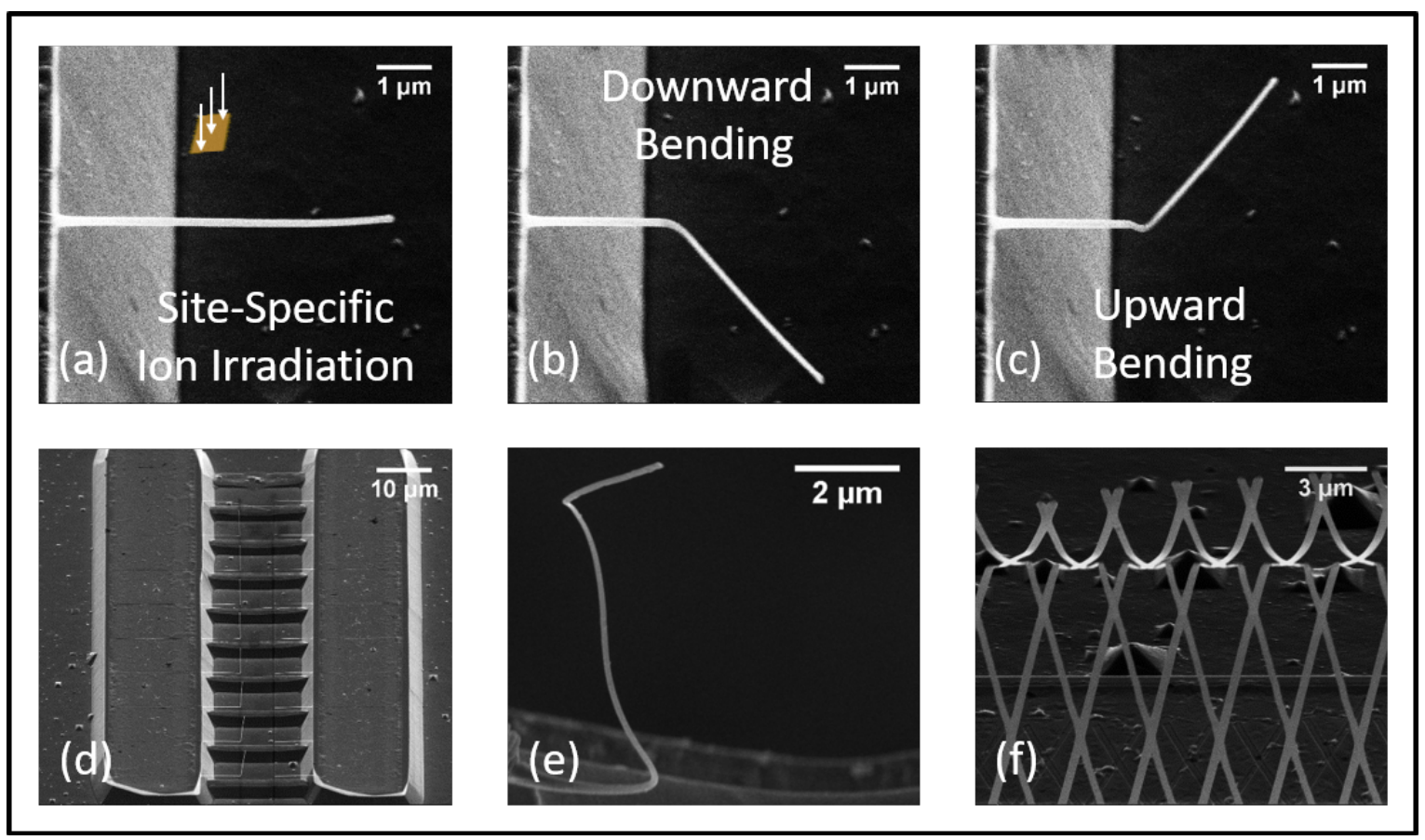

Figure 1. Ion induced bidirectional bending for controlled manipulation at nanoscale: SEM image showing (a) freestanding Si NW realized with wet etching of ion implanted $\mathrm{Si}$; site-specific ion irradiation of suspended NW developing (b) downward, (c) upward bending at low, high ion doses respectively; (d) an array of vertically bent NWs, (e) NW bent in two alternative directions, (f) a curved 3D mesh nanostructure showing controlled manipulation of nanostructures through in-situ ion irradiation 